\title{
Construction and application of comprehensive early warning system for coal and gas outburst in Xinjing Coal Mine
}

\author{
Yunlong Zou ${ }^{1,2,3, *}$ \\ ${ }^{1}$ State Key Laboratory of the Gas Disaster Detecting, Preventing and Emergency Controlling, Chongqing, 400037, China \\ ${ }^{2}$ Chongqing Research Institute of China Coal Technology and Engineering Group Crop., Chongqing, 400037, China \\ ${ }^{3}$ Chongqing University, Chongqing, 400044, China
}

\begin{abstract}
In order to further strengthen the prevention and control of coal and gas outburst in Xinjing Coal Mine, the online comprehensive analysis and early warning index system and rules of coal and gas outburst suitable for Xinjing Coal Mine were studied. Based on the corresponding early warning computer system and guarantee mechanism, a comprehensive early warning system for coal and gas outbursts in Xinjing Coal Mine was established, realizing real-time intelligent early warning of outburst dangers in working faces. The system realizes the standardization and dynamic management of outburst prevention information at the working face, as well as the real-time dynamic update and sharing of outburst prevention information, which improves the efficiency of mine outburst prevention management and the level of mine safety. The actual application of the system in Xinjing Coal Mine shows that the system can provide an effective reference for the comprehensive early warning of outbursts in other outburst mines of Yangquan Coal Industry Group.
\end{abstract}

\section{Introduction}

Coal and gas outburst poses a serious threat to the safety of mine production [1-2]. The primary link of coal and gas outburst prevention is to judge the possibility and intensity of outburst based on the precursor information of the outburst [3]. A certain predictive index can only indirectly and partially reflect a certain aspect of the outstanding risk, and there is no early warning of the outstanding risk and its severity from multiple directions and perspectives, which has considerable limitations [48]. At the same time, with the development of computer technology and information technology, the informatization of coal mine safety management will also be an irreversible development trend [9-13]. Therefore, with the help of computer technology and information technology, a coal and gas outburst warning system suitable for the specific conditions of the mine is established to realize the information management of mine safety and the early warning of mine outburst disasters. This will be one of the important technical means for coal mines to effectively prevent and control outstanding disasters and ensure coal mine production safety in the future.

At present, the coal and gas outburst disasters in the mines of Yangquan Group are serious, and the outburst has become the main factor restricting the safe and efficient production of the outburst mines of the group. Taking the Xinjing Coal Mine of Yangquan Coal Group as the pilot unit, aiming at the problems existing in the current gas prediction and forecasting in the mine, comprehensive information such as gas geology, gas emission, and roof pressure in the outburst mine is integrated, there is an urgent need to enhance the mine's ability to prevent coal and gas outburst disasters and improve the safety management level for Xinjing Company to, so as to promote the application of experience to other outburst mines of Yangquan Coal Group.

\section{Research on early warning index system of coal and gas outburst}

The research is the prerequisite and foundation for the implementation of the entire early warning system project. It is the development of early warning system software and provides a technical basis for the realization of comprehensive, intelligent, advanced and dynamic early warning of coal and gas outbursts in coal mines [14-15]. The system mainly focuses on the research of comprehensive early warning technology [16]. The index system framework of the entire early warning system (as shown in Figure 1) mainly includes six aspects: gas geology, mining impact, daily prediction, gas emission, mine pressure, and outburst prevention measures. Under the guidance of this framework, examine the specific early warning indicators and rules suitable for Xinjing Company, and determine the critical value of each early warning indicator and its relationship with the degree of outstanding danger, and finally establish a prominent early warning indicator system that is in line with Xinjing Company's actual situation.

\footnotetext{
* Corresponding author: 2010152@cqceteg.com
} 


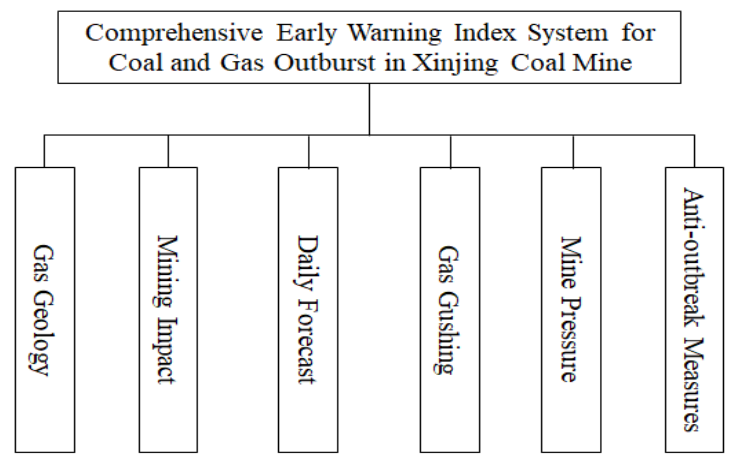

Figure.1 Framework of comprehensive early warning indicator system for coal and gas outburst in Xinjing Coal Mine

Early warning rules should include status warning rules and trend warning rules. Among them, the state early warning rules are the rules set to reflect the current state of the work face with outstanding hazards, and the trend early warning rules are mainly the rules formulated to reflect the changing trend of the prominent hazards in the front of the work face.

Xinjing's outstanding early warning rules are divided into 6 categories, a total of 57, including 11 gas geological categories, 26 mining impact categories, 7 daily forecast categories, 4 gas emission categories, 6 mine pressure categories, and 3 outburst prevention measures. In the production process, the value of each early warning indicator reflects the state of the early warning elements of the working face [3].

\section{Construction of a comprehensive early warning system for coal and gas outbursts}

The coal and gas outburst early warning computer system is a large-scale computer program based on the combination of GIS, C/S and B/S. Through the outburst early warning module, the system analyzes the coal and gas outburst early warning data in the comprehensive early warning data server, and sends out early warning information according to the analysis results [17]. According to the characteristics and related requirements of the outburst prevention and control work in Xinjing Coal Mine, the comprehensive early warning system for coal and gas outburst in Xinjing Coal Mine includes 7 subsystems and 1 outburst early warning website. Seven of the subsystems are mainly geological measurement management system, gas geological dynamic analysis system, gas outburst dynamic management and analysis system, gas emission dynamic analysis system, mining progress management system, mine pressure monitoring and early warning system, and gas outburst comprehensive early warning platform [3].

The stable operation of the coal and gas outburst early warning system is the prerequisite for accurate early warning. After the software adaptability adjustment and preliminary system test are completed, the system functions are gradually improved and the overall operation is stable. The specific performance is as follows:
(1) The hardware of the early warning system runs reliably. During the inspection, the early warning server and client computers were operating completely normally, and there were no machine failures such as crashes or abnormal shutdowns. After on-site testing, the UPS power supply can guarantee the normal operation of the early warning server for 20 minutes in the event of an abnormal power failure. The SMS publishing platform can promptly and accurately publish the warning result information.

(2) The early warning system software responds quickly. After many tests, the various subsystems of the early warning system open the local mine map generally within $10 \mathrm{~s}$. When the office network is normal, the time to open the server mine map directly is within $15 \mathrm{~s}$. Data synchronization and early warning analysis can generally be completed within 5 minutes under normal network conditions.

(3) The error rate of the entire early warning system is low. When the user operates and maintains the early warning system in accordance with the "Xinjing System Early Warning System Job Responsibility" and related operational requirements, the early warning system can calculate early warning indicators in a timely and accurate manner and release the early warning results in a timely manner. The system runs continuously and stably for longer than 3 months.

\section{Application effect analysis}

\subsection{Follow-up inspection of daily early warning effect at work face}

According to the relevant information, during the inspection period, there were two major outburst dangers in the 3\# Coal South Eighth Lane in Lunan Second District, one occurred on February 2, and the K1 value was predicted to reach $1.28 \mathrm{~mL} / \mathrm{g} \cdot \mathrm{min} 1 / 2$, the thickness of the soft layer is $0.8 \mathrm{~m}$.

The trend pre-alert for the zero o'clock shift on January 30 is "orange", and the trend warning for the shift at four o'clock on February 1 will be upgraded to "red", and the state warning for the eight o'clock shift on February 2 will be upgraded to "danger", and the trend warning will be "orange". The K1 forecast on duty exceeded the standard, and the triggering factors of this early warning process were all gas gushing (Figure 2). The early warning results were accurate and had good forecasting advancement (the $\mathrm{K} 1$ value was 2 days ahead). In addition, on February 2nd, the thickness of the soft layer exceeded the warning threshold of $0.3 \mathrm{~m}$. 


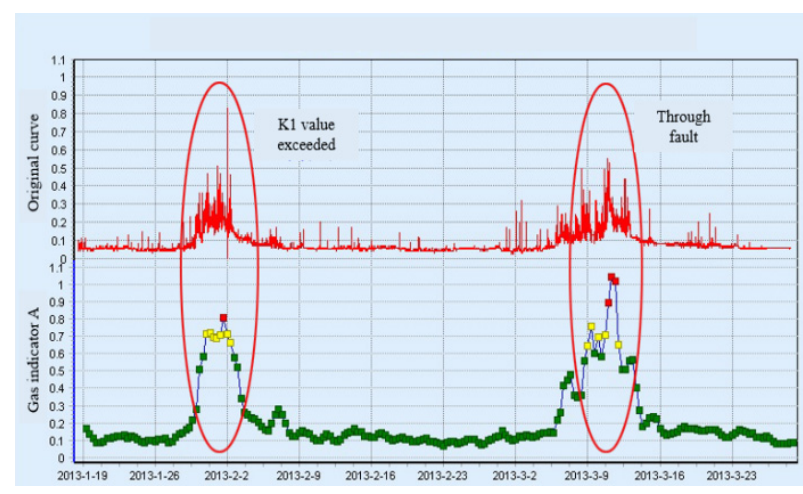

Figure.2 Early warning results of gas emission in Nanba main lane

The pre-warning of the eight o'clock shift on March 7 was "orange". And at 8 o'clock on March 10, the trend warning was upgraded to "red" and the status warning was "threatening", indicating that the working face has entered the structural influence range and the structure will be revealed soon. By the start of the zero o'clock shift on March 9, it gave an "orange" warning from the gas gushing angle, and then the zero o'clock shift was upgraded to a "red" warning on March 11. This situation shows that under the action of geological structure, gas gushing is abnormal. In this early warning process, two aspects of gas geology and gas gushing were carried out to warn the outstanding hazards in front of the work. The law of occurrence of outstanding hazards was completely consistent with theoretical research and on-site conditions. This further shows that the early warning system has good advancement and reliability.

\subsection{Analysis of typical daily warning results}

The daily forecast of No. 8 Coal East No.1 Main Lane in Lunan No. 1 District exceeded the standard at 8 o'clock on July 4th. K1 reached $1.49 \mathrm{~mL} / \mathrm{g} \bullet \mathrm{min} 1 / 2$, and the $\mathrm{K} 1$ value was too large, indicating that there is a greater risk of outburst in front of the work. The trend warning result of the zero o'clock shift on July 2 was "orange", and the status warning result was "normal". The trend warning result of the eight o'clock shift on July 2 remained "orange", and the status warning result was upgraded to "threatening". However, because the predicted K1 value of the working face did not exceed the standard (the index value is $0.35 \mathrm{~mL} / \mathrm{g} \bullet \mathrm{min} 1 / 2$ ), and there is no obvious gas dynamic phenomenon, the working face continued to move forward until the zero o'clock shift on July 3, the early warning result is upgraded to "red".

On the same day, the K1 value still did not exceed the standard (the index value was $0.35 \mathrm{~mL} / \mathrm{g} \bullet \mathrm{min} 1 / 2$ ), and there was no dynamic phenomenon, so the tunneling operation was still carried out according to the normal procedure until the $\mathrm{K} 1$ value of the eight o'clock shift on July 4 exceeded the standard. The work face stopped work and construction measures were taken. Subsequently, the results of the trend warning and status warning of the shift at 8 o'clock on July 5 became "normal".

The triggering factor of this alarm was abnormal gas gushing, and it issued a prominent hazard warning 2 days ahead of Xinjing's daily forecast of K1 value, indicating that the early warning is well advanced and can leave a sufficient safety distance for the implementation of antioutbreak measures. At the same time, from July 1st to July 3rd, the gas emission index showed a gradually increasing pattern of change (Figure 3), which was in full compliance with the general law of "zone and zone" distribution of prominent hazards. After July 4th, with the gradual elimination of the prominent danger of the working face, the warning level has also been reduced, which fully shows that the warning results have high reliability.

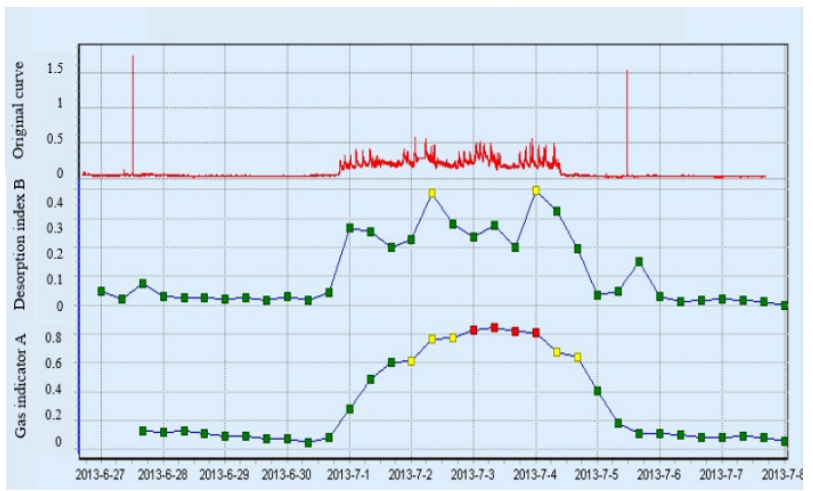

Figure.3 Early warning results of Dongyi main lane

The early warning result of Nanba main lane is shown in Figure 4. It can be seen from Figure 4 that the trend warning result of the early warning system at the eight o'clock shift on July 28 was "orange", and the trend warning result of the zero shift was upgraded to "red" on July 29, and the K1 value of the eight o'clock shift predicted that the K1 value exceeded the standard on July 30 . This time, the early warning system has advanced the $\mathrm{K} 1$ value by 2 days from the angle of gas emission to provide early warning of the prominent danger in front of the Nanba main lane excavation work.

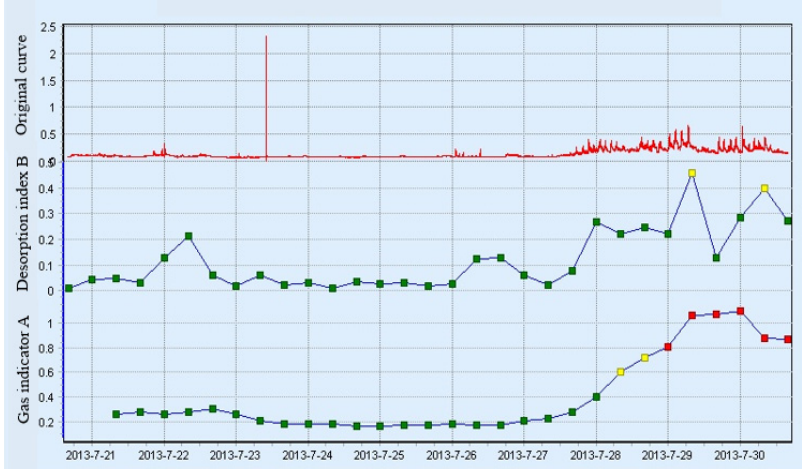

Figure 4. Early warning results of Nanba main lane

\section{Conclusion}

(1) Based on the actual site data and data of Xinjing Coal Mine, comprehensively analyzed the impact on the outburst hazard of the mining face of Xinjing Coal Mine, and established consideration of gas geology, mining impact, daily prediction, gas emission, mine pressure and Six factors including anti-outburst measures are applicable to Xinjing's coal and gas outburst online 
comprehensive analysis and early warning indicator system and rules.

(2) According to the characteristics and related requirements of the outburst prevention and control work of Xinjing Coal Mine, a comprehensive early warning system for coal and gas outbursts of Xinjing Company was established. The system mainly includes the geological survey management system, the gas geological dynamic analysis system, and the outburst prevention dynamic management and analysis.

(3) The comprehensive early-warning system for coal and gas outburst constructed in Xinjing Coal Mine has been inspected on site application effects and showed that the software and hardware of the comprehensive earlywarning system can ensure the continuous and stable operation of the early-warning system, as well as timely and accurate release of early-warning results.

\section{Acknowledgments}

The study was supported by the Special Fund Project for Safety Production in Chongqing (Coal Development) (2020B182[5985]20015501), Chongqing Research Institute Independence Project (202001120).

\section{References}

1. Si Hu, Zhao Jiannan, Hu Qianting. Analysis of causes of coal and gas outburst accidents based on big data theory[J]. Journal of Xi'an University of Science and Technology,2018,38(04):515-522+537.

2. Jiang Anfei, Sun Dongling, Liu Yanbao, et al. Study on the effect of gas pressure on the propagation of coal and gas outburst shock wave [J]. Mining Safety and Environmental Protection, 2021, 48(02): 18-22.

3. Zhao Xusheng, Sun Haitao, Cao Jie, et al. Applications of online integrated system for coal and gas outburst prediction A case study of Xinjing Mine in Shanxi China $[\mathrm{J}]$. Energy Science and Engineering,2020,8(6): 1980-1996

4. Zhao Xusheng, Ma Guolong, Qiao Wei, et al. Coal and gas outburst warning index system based on fault tree analysis [J]. Mining Safety and Environmental Protection, 2019, 46(03): 37-43.

5. Ning Xiaoliang. Research status and development trend of early warning technology for coal and gas outburst $[\mathrm{J}]$. Industry and Mine Automation, 2019, 45(08): 25-31+37.

6. Cao Kang, Li Zhonghui, Yu Desheng, et al. Research on precursor characteristics and comprehensive early warning of coal and gas outbursts in driving face [J]. Coal Science and Technology, 2020, 48(11): 147-152.

7. Song Dazhao, He Xueqiu, Qiu Liming, et al. Research on dynamic real-time monitoring and early warning technology of regional and local outburst hazards[J]. Coal Science and Technology, 2021, 49(05): 110-119.

8. Xu Xuezhan. Continuous prediction technology and application of coal and gas outburst based on gas emission characteristics $[\mathrm{J}]$. Energy and Environmental Protection, 2021, 43(01): 1-7.

9. $\mathrm{Pu}$ Yang. High-yield and high-efficiency mine coal and gas outburst far and near field collaborative prediction technology[J]. Coal Mine Safety, 2020, 51(07): 70-74+80.

10. Ning Xiaoliang. Coal and gas outburst warning technology based on big data[J]. Mining Safety and Environmental Protection, 2020, 47(04): 51-56.

11. Long Nengzeng, Yuan Mei, Wang Guanliang, et al. Research on real-time early warning of coal and gas outburst based on data mining[J]. China Mining Industry, 2020, 29(11): 88-93+99.

12. Yao Yahu. Application of multi-parameter outburst warning technology based on gas geological characteristics in new coal mines [J]. Mining Safety and Environmental Protection, 2021, 48(03): 94-99.

13. Cheng Xiaoyang, $\mathrm{Pu}$ Yang, Song Zhiqiang. Application of outburst warning system based on gas monitoring data source in Xiayukou Coal Mine [J]. Mining Research and Development, 2021, 41(07): 161-165.

14. Niu Jufen, Cheng Wuyi. Construction of early warning index system for coal and gas outburst based on time dimension[J]. Coal Engineering, 2012(05): 96-98.

15. Tan Guowen. Research on the determination method of coal and gas outburst warning indicators based on gas emission characteristics in Wangjiazhai Coal Mine [J]. Mining Safety and Environmental Protection, 2015, 42(03): 34-37.

16. Che Yuheng. Application of multi-source information intelligent early warning system for coal and gas outburst [J]. IOP Conference Series: Earthand Environmental Science, 2021,781(4): 042027.

17. Ning Xiaoliang. Construction and application of comprehensive early warning system for coal and gas outburst in Wangjiazhai Coal Mine[J]. Energy Technology and Management, 2014, 39(02): 5-7. 